Case report

\title{
Pigmented villonodular synovitis in multiple joints \\ Occurrence in a child with cavernous haemangioma of lip and pulmonary stenosis
}

\author{
J. LESZCZYNSKI,* J. R. HUCKELL, J. S. PERCY, J. C. LERICHE, AND B. C. LENTLE \\ From the Rheumatic Disease Unit, the Division of Orthopaedic Surgery, and the Department of Pathology, \\ University of Alberta; and the Department of Nuclear Medicine, W.W. Cross Cancer Institute, \\ Edmonton, Alberta
}

\begin{abstract}
Leszczynski, J., Huckell, J. R., Percy, J. S., LeRiche, J. C., and Lentle, B. C. (1975). Annals of the Rheumatic Diseases, 34, 269-272. Pigmented villonodular synovitis in multiple joints. Occurrence in a child with cavernous haemangioma of lip and pulmonary stenosis. A case is presented of pigmented villonodular synovitis involving three joints in a 7-yearold girl. The diagnosis was confirmed at surgery and by histology. The patient also exhibited a haemangioma of the upper lip and a congenital pulmonary stenosis of mild degree. Subtotal synovectomy of the right knee and of both ankles was performed. The lesion recurred in both ankles after 6 months. Review of the literature failed to reveal any previous report of multiple joint involvement by pigmented villonodular synovitis in childhood and it appears that simultaneous involvement of three joints has not previously been described. Scintiscanning with ${ }^{99 m}$ Tc stannous pyrophosphate showed increased vascularity of the involved joints immediately after injection, but no increased osteoblastic activity was seen on the delayed scan. This radionuclide scanning technique is therefore helpful in distinguishing pigmented villonodular synovitis from other arthropathies.
\end{abstract}

Pigmented villonodular synovitis is an unusual condition of unknown aetiology. It has been suggested that it is the result of recurrent intra-articular haemorrhage and attention has been drawn to the histological similarity between pigmented villonodular synovitis and the synovial changes which occur in haemophilia (Jaffe, Lichtenstein, and Sutro, 1941). Similar changes have been induced by intra-articular injection of autogenous blood in dogs and it has been stated that 'the presence of blood in joints is essentially for the production of pigmented villonodular synovitis' (Young and Hudacek, 1954).

The condition has been described in the knee joint in three children who also had intra-articular haemangiomata of the affected joint (Bobechko and Kostuik, 1968). Three adult patients have been reported in each of whom the disease was present in two joints (Byers, Cotton, Deacon, Lowry, Newman, Sissons, and Thomson, 1968; Gehweiler and Wilson,
1969), but there are no reports of multiple joint involvement in childhood.

The present case is of special interest because it is the first report of the simultaneous occurrence of pigmented villonodular synovitis in three joints in a child. A point of potential importance is that a radionuclide angiogram followed by a static bone scan suggests that the lesions are primarily vascular and not inflammatory.

\section{Case report}

The patient, an undersized 7-year-old girl with a bone age of $4 \frac{1}{2}$ years, was admitted to the University of Alberta Hospital complaining of recurrent, painful swelling in the right knee of 9 months' duration. Several aspirations of the affected knee had yielded uniformly blood-stained fluid. There was no history of antecedent injury but the child had had an eventful past history with 7 previous hospital admissions because of pulmonary stenosis and respiratory 


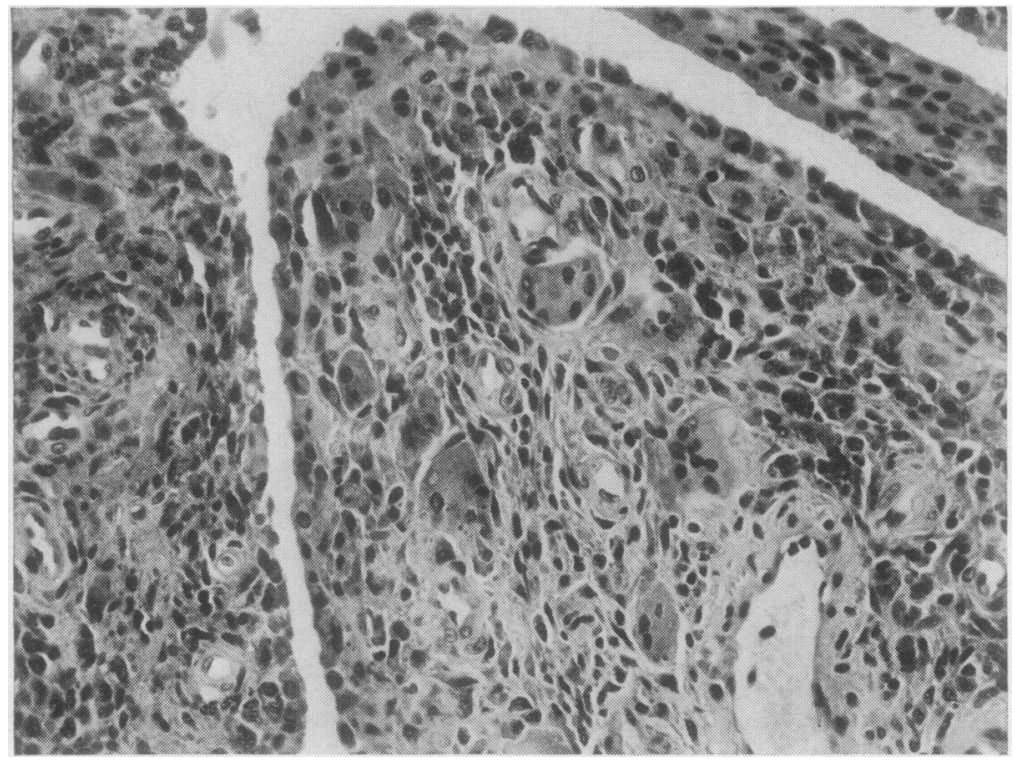

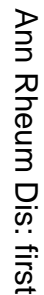

FIG. 1 Histological appearance of 0 excised synovium showing giant $\bar{C}$ cells, haemosiderin-laden macro- $\overline{\bar{\omega}}$ phages, and foam-laden micro- $\widehat{\nabla}$

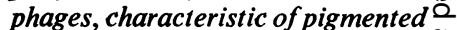
villonodular synovitis

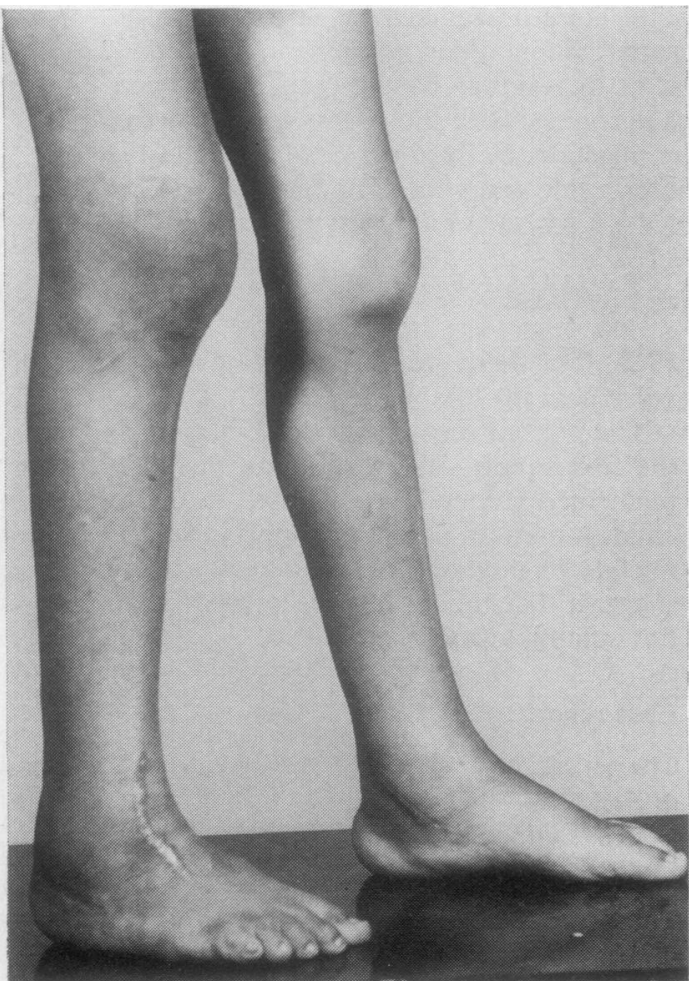

FIG. 2 Recurrent pigmented villonodular synovitis in right knee and both ankles

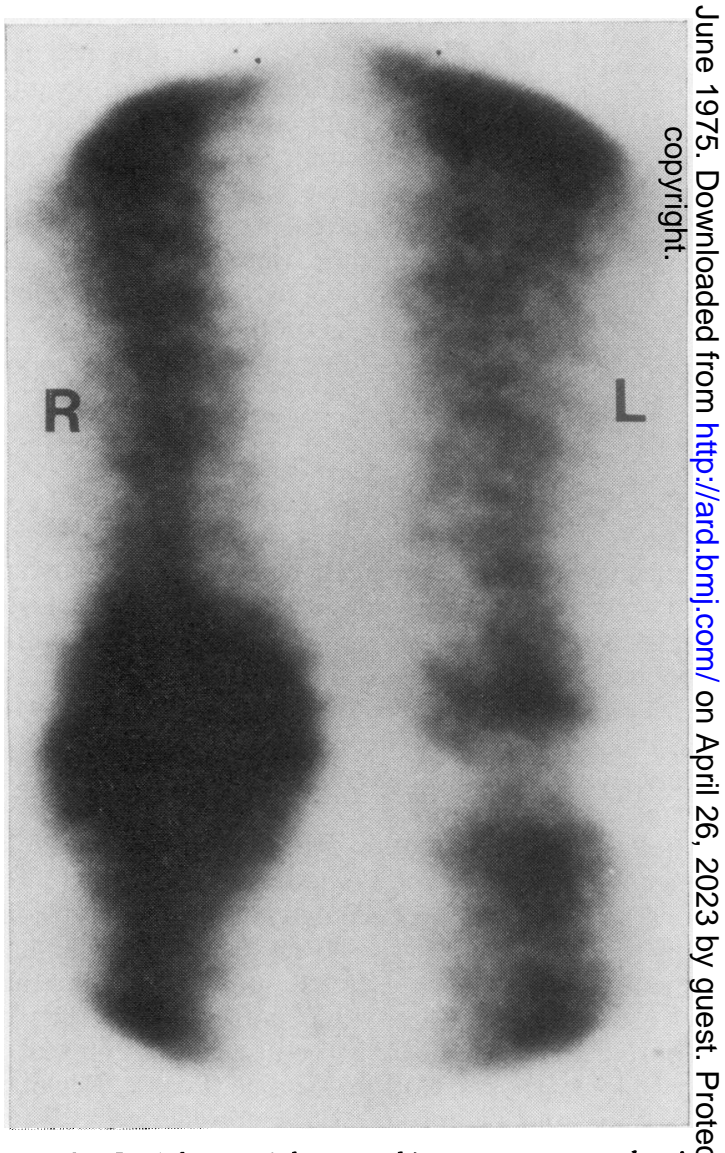

FIG. 3 Initial scintiphotographic appearance showin markedly increased vascularity in the right knee (Picken Dynacumera 20) 
infection. There was no family history of joint disease. Examination revealed a boggy swelling of the right knee and both ankles. A full range of movement was present in all joints. Other positive findings included a haemangioma of the upper lip and a palpable spleen. Slight cyanosis of lips and nails was present but there was no finger clubbing. A grade III/IV systolic murmur was heard mainly over the pulmonary area consistent with the mild pulmonary valvular stenosis previously shown by cardiac catheterization.

With the exception of the finding of a mild iron deficiency anaemia, the routine laboratory investigations were normal. $X$-rays of the affected joints showed soft tissue thickening, together with some apparent increase of joint space in both ankles. No bony abnormalities were seen. Aspiration of the right knee again yielded bloody synovial fluid.

The possibility of intra-articular haemangiomata was considered because of the angioma on the upper lip and therefore an aortic angiogram was performed. There was no evidence of any arteriovenous malformation, but increased vascularity was observed in what appeared to be hypertrophic synovium in both ankles and in the right knee.

Subsequent arthrotomy of the right knee showed thickened, brownish-red synovium with sessile and pedunculated villous overgrowths. This was excised. The histological appearances of the synovium are shown in Fig. 1. No vascular abnormality was observed and there was no evidence of malignancy. Subsequent arthrotomy and synovectomy performed on both ankles showed synovium of similar clinical appearance lining joint cavities and extending along the tendon sheaths adjacent to the joint.

Pathological examination of the synovium of both ankles and the right knee showed similar gross and microscopical appearances. The synovial surface was covered by masses of filiform and nodular deeply pigmented masses. Within such excrescences large numbers of pigment-containing macrophages and small clusters of histiocytes containing lipid were seen. Many giant cells, some of which contained pigment, were also present. In addition, there was a proliferation of synovial cells and the entire lesion was infiltrated by a chronic inflammatory infiltrate showing a predominance of lymphocytes in other areas while some areas showed clusters of plasma cells. Within the synovium and the perisynovial connective tissues, vascular proliferation and dilatation were encountered, although no true haemangioma could be identified. 18 months after operation the patient showed major recurrence of the condition in both ankles and in the right knee (Fig. 2).

A bone scintiscan was obtained using $6 \mathrm{mCi}{ }^{99} \mathrm{~m}_{\text {techne- }}$ tium stannous pyrophosphate (Mallinckrodt). At the time of injection of the radionuclide into the arm, serial gamma camera scintiphotographs of the knees were obtained with a Picker Dynacamera 2C gamma camera. Immediate scans were also obtained to an information density of 2000 counts $/ \mathrm{cm}^{2}$.

Delayed bone scintiscans were then obtained $3 \frac{1}{2}$ hours after using an Ohio Nuclear Dual $5^{\prime \prime}$ proble rectilinear scanner operated in a 5:1 minification mode.

The initial scintiscan (Fig. 3) reflects the vascularity of a lesion and in this patient marked asymmetry of the vascular supply to the knees was evident, it being greatly increased on the right. This increased vascularity enveloped the entire knee and ankle regions while the delayed scans, reflecting osteoblastic activity more particularly, showed increased uptake at the open epiphyses with much less marked asymmetry at the knees and only a relatively small contribution from residual vascular pool and increased osteoblastic reaction.

\section{Discussion}

The general incidence of pigmented villonodular synovitis is said to be $1 \%$ of all patients presenting with joint complaints (Clark, 1958). Pigmented villonodular synovitis is distinctly an unusual occurrence in children. Three patients, aged 4, 5, and 10 years, respectively, have been described with the condition. A recent review showed that 13 of 78 cases occurred in the second decade, the youngest patient being 11 years (Byers and others, 1968). From these same data it is apparent that pigmented villonodular synovitis occurs principally in young adults with a peak incidence in the fourth decade.

Two distinct histological types of pigmented villonodular synovitis are described (Byers and others, 1968; Chung and Janes, 1965; Jaffe and others, 1941), the nodular and the diffuse. The former is predominantly found in the flexor tendon sheaths of the fingers but also occurs in larger joints, such as the knee where a pedunculated tumour may cause instability and locking. The diffuse form occurs most frequently in the knee but other large joints may also be involved. This form is characteristically monarticular and is associated with bloody effusion (Byers and others, 1968).

Three histologically confirmed cases of the diffuse type of pigmented villonodular synovitis involving two joints have been reported in adults (Byers and others, 1968; Gehweiler and Wilson, 1969; Greenfield and Wallace, 1950). In one case both knees were involved (Greenfield and Wallace, 1950), in another both ankles (Byers and others, 1968), and in a third a hip and a knee (Gehweiler and Wilson, 1969). There is also brief mention of bilateral knee involvement in three sibs of one family (Kelikian and Lewis, 1949), however no statement is made as to whether this was a histological diagnosis or one based on clinical observation alone. In this context it is of interest that both parents and eight sibs of our patient were examined and found to have no evidence of joint disease. It appears that the present case where three joints were involved and the diagnosis was confirmed histologically is the first of its kind. Similarly, no mention can be found in the literature of pigmented villonodular synovitis affecting more than one joint in childhood.

Three cases of pigmented villonodular synovitis of the knee in children were all associated with haemangiomata (Bobechko and Kostuik, 1968). These were both cutaneous (one localized and two involving the 
entire lower limb) and intra-articular (confirmed histologically and on arteriography). In this connexion it is interesting to note that intra-articular haemangioma not associated with pigmented villonodular synovitis was found to coexist frequently with cutaneous haemangioma ( $27 \%$ in one series-Coventry, Harrison, and Martin, 1966). Our patient did not have any arteriographic or histological evidence of haemangioma in any of the involved joints, although she did have a squamous haemangioma of the upper lip. It is possible that small capillary haemangiomata may have been present, although meticulous histological search failed to reveal their presence.

We know of no other reports of the use of scintiscanning techniques in pigmented villonodular synovitis. The appearance of increased vascularity on the initial scan, with no evidence of increased osteoblastic activity on the delayed bone scintiscan, is in marked contrast to the findings in other arthropathies in which there is considerable increase in synovial permeability or osteoblastic activity (Maxfield, Weiss, and Shuler, 1972; Berry, Browett, Huskisson, Bacon, and Willoughby, 1973; Desaulniers, Fuks, Hawkins, Lacourciere, and Rosenthall, 1974; Lentle, Percy, and Russell, 1975). This suggests that pigmented villonodular synovitis is primarily a vascular disorder of the synovium, and that the other changes are secondary o to intra-articular haemorrhage.

This case serves to emphasize the diagnostic importance of frankly blood-stained synovial fluid in pigmented villonodular synovitis if haemophilia and trauma have been excluded.

\section{References}

Berry, H., Browett, J. P., Huskisson, E. C., Bacon, P. A., ANd Willoughby, D. A. (1973) Ann. rheum. Dis., 32, 95 (Measurement of inflammation I. Application of technetium clearance to rheumatoid arthritis and animal models)

Bobechko, W. P., AND Kostuik, J. P. (1968) Canad. J. Surg., 11, 480 (Childhood villonodular synovitis)

Byers, P. D., Cotton, R. E., Deacon, O. W., Lowry, M., Newman, P. H., Sissons, H. A., AND Thomson, A. D. (1968) J. Bone Jt. Surg., 50B, 290 (Diagnosis and treatment of pigmented villonodular synovitis)

Chung, S. M. K., AND JANeS, J. M. (1965) Ibid., 47A, 293 (Diffuse pigmented villonodular synovitis of the hip joint)

Clark, W. S. (1958) Bull. Rheum. Dis., 8, 161 (Pigmented villonodular synovitis)

Coventry, M. B., Harrison, E. G., AND MARTIN, J. F. (1966) J. Bone Jt. Surg., 48A, 1350 (Benign synovial tumors of the knee: a diagnostic problem)

Desaulniers, M., Fuks, A., Hawkins, D., Lacourciere, Y., and Rosenthall, L. (1974) J. Nucl. Med., 15, 417 (Radiotechnetium polyphosphate joint imaging)

GeHWEILER, J. A., AND WiLsON, J. W. (1969) Radiology, 93, 845 (Diffuse biarticular pigmented villonodular synovitis)

Greenfield, M. M., ANd Wallace, K. M. (1950) Ibid., 54, 350 (Pigmented villonodular synovitis)

JAFFe, H. L., Lichtenstein, L., AND SuTro, C. J. (1941) Arch. Path., 31, 731 (Pigmented villonodular synovitis, bursitis and tenosynovitis)

KelikiAn, H., AND LeWIS, E. L. (1949) Radiology, 52, 465 (Arthrograms)

Lentle, B. C., PerCy, J. S., AND Russell, A. S. (1975) (Bone scintiscanning in osteoarthritis) (In preparation)

Maxfield, W. S., Weiss, T. E., AND Shuler, S. E. (1972) Semin. Nucl. Med., 2, 50 (Synovial membrane scanning in arthritic disease)

Young, J. M., AND Hudacek, A. G. (1954) Amer. J. Path., 30, 799 (Experimental production of pigmented villonodular synovitis in dogs) 